\title{
Plantar Pressure Differences Between Nordic Walking Techniques
}

\author{
by \\ Alberto Encarnación-Martínez', Ángel Gabriel Lucas-Cuevas², \\ Pedro Pérez-Soriano ${ }^{2}$, Ruperto Menayo ${ }^{1}$, Gemma María Gea-García ${ }^{1}$
}

\begin{abstract}
High plantar pressure has been associated with increased risk of injury. The characteristics of each physical activity determine the load on the lower limbs. The influence of Nordic Walking (NW) technique on plantar pressure is still unknown. The aim of this study was to analyze the differences between plantar pressure during NW with the Diagonal technique (DT) versus Alpha technique (AT) and compare them with the pressure obtained during normal walking $(W)$. The normality and sphericity of the plantar pressure data were checked before performing a two-way repeated measures ANOVA in order to find differences between speeds (preferred, fast) and the gait (NW, W) as within-subject factors. Then, a t-test for independent measures was used to identify the specific differences between NW techniques. The strength of the differences was calculated by means of the effect size (ES). The results demonstrated that during NW with AT at preferred speed the pressure was lower under the Calcaneus, Lateral Metatarsal and Toes compared to the DT group ( $p=0.046, E S=1.49 ; p=0.015, E S=1.44 ; p=0.040, E S=1.20$, respectively). No differences were found at the fast speed ( $p>0.05)$. Besides the increase in walking speed during $N W(p<0.01)$, both technique groups showed lower pressure during NW compared to $W$ under the Hallux and Central Metatarsal heads $(F$ $=58.321, p=0.000, E S=2.449 ; F=41.917, p=0.012, E S=1.365$, respectively). As a practical conclusion, the AT technique may be the most effective of the NW techniques at reducing plantar pressure while allowing NW practitioners to achieve the physiological benefits of NW.
\end{abstract}

Key words: poles, biomechanics, foot, loading.

\section{Introduction}

The popularity of Nordic Walking (NW) has increased considerably in recent years due to the numerous health benefits associated with its regular practice. Including NW in a programme of physical activity (3-4 days a week for several months) provides long-term benefits for health including an improvement of $40 \%$ in muscle endurance in the upper limbs, as well as a reduction in neck and shoulder pain (FigardFabre et al., 2010).

From a physiological perspective, NW increases the energy expenditure compared to

normal walking (W) although differences in the ratings of perceived exertion have not been observed between these activities (Church et al., 2002). Moreover, the extra support provided by the poles in NW leads to improved stability which makes NW a recommendable activity for the elderly and also for people with balance and stability deficits. When walking at matched speeds, the oxygen uptake, heart rate, expiratory volume, energy expenditure, and lactic acid levels are increased during NW when compared to $\mathrm{W}$ (Pérez-Soriano et al., 2014). As special populations

1 - Department of Sport and Physical Education, Catholic University of Murcia, Spain.

2 - Department of Physical Education and Sport, University of Valencia, Spain. 
(e.g. the elderly, obese and diabetic patients) face greater difficulties in carrying out physical activity on a regular basis, the inclusion of an activity similar to walking with larger physiological and cardiovascular benefits such as NW seems a very appealing alternative.

From a biomechanical perspective, NW increases stride length, walking speed and muscle activity in the upper limbs compared to W (Hagen et al., 2011; Stief et al., 2008). In addition, whereas one study found lower ground reaction forces (GRF) during NW compared to W (Schwameder and Ring, 2006), the majority of the studies have observed greater GRF during NW (Brunelle and Miller, 1998; Encarnación-Martínez et al., 2015; Hagen et al., 2011; Stief et al., 2008). On the other hand, there are very few studies analyzing plantar pressure during NW. In this sense, the PérezSoriano et al.'s (2011) study was the first to observe that NW reduced the plantar pressure by $50 \%$ under the central metatarsals. Moreover, these authors demonstrated that performing NW on a regular basis also led to a $40 \%$ reduction of plantar pressure under the central metatarsals even during $\mathrm{W}$ without poles. Similarly, Hudson (2014) observed that the use of poles during W reduced pressure under the metatarsal heads by $24 \%$ and concluded that this activity was easy to learn and could be very beneficial for people at a greater risk of developing plantar ulcers.

Elevated plantar pressure is considered a risk factor in the development of plantar ulcers in the diabetic (Menz and Morris, 2006) and obese (Vela et al., 1998) population. In that sense, Perry et al. (1995) demonstrated that the highest pressure in the neuropathic diabetic foot was under the 2nd metatarsal head, followed by the lateral metatarsal heads, 1st metatarsal head, and the heel. Thus, it is difficult to provide clinical magnitude of the pressure values considering the heterogeneity of the protocols employed (assessment tools, testing speeds, the features of the subjects, etc.), although the existence of higher plantar pressure in the zones of medial and central metatarsal heads has been correlated with a risk factor of getting injured (Lyons et al., 2012). Therefore, strategies that aim to reduce pressure in the foot are necessary for these populations.

In this sense, even the use of low-impact activities such as walking in programmes of physical activity for obese patients has been observed to increase plantar pressure in four of the ten areas analyzed, especially under the heel, metatarsal heads and lateral of the foot (De Castro et al., 2014). Hudson (2014) demonstrated that by modifying the ground contact technique during walking with poles it was possible to reduce plantar pressure under the metatarsals heads, although this reduction was not observed under the hallux. However, Pérez-Soriano et al. (2011) did observe a reduction of plantar pressure under this area in amateur Nordic walkers. The lack of pressure differences under the hallux observed in the Hudson's study could be due to the fact that Hudson (2014) analyzed the effect of different coordination patterns on plantar pressure during walking with poles and no condition was NW, whereas Pérez-Soriano et al. (2011) investigated the differences between NW performed by experienced practitioners and $\mathrm{W}$.

To the best of our knowledge, it is still unknown how the different NW techniques influence plantar pressure. In this sense, there are mainly two different NW techniques: the Diagonal technique (DT) and Alpha technique (AT). Whereas in the DT athletes tend to bend their trunk slightly forward and perform larger movements with their upper limbs, in the AT athletes have a more upright position of the trunk throughout the movement with less range of motion of the upper limbs.

The objective of this study was to compare the plantar pressure distribution between two different NW techniques and $\mathrm{W}$ at different speeds in amateur Nordic walkers. The following hypotheses were tested:

$\mathrm{H} 1$ : Increases in walking speed will lead to greater plantar pressure.

$\mathrm{H} 2$ : NW will reduce the plantar pressure compared to $\mathrm{W}$.

H3: The mechanical differences between NW techniques (Alpha, Diagonal) will lead to different plantar pressure distribution.

\section{Material and Methods}

\section{Participants}

Twenty-seven physically active sport science students were randomly assigned to one of the training groups: Alpha Technique (AT, $\mathrm{n}=$ 15 , age $=20.4 \pm 1.6$ years; body mass $=75.5 \pm 10.2$ $\mathrm{kg}$; body height $=179.1 \pm 8.8 \mathrm{~cm}$ ) or Diagonal Technique $(\mathrm{DT}, \mathrm{n}=12$, age $=19.3 \pm 0.5$ years; body 
mass $=72.1 \pm 7.6 \mathrm{~kg}$; body height $=177.6 \pm 5.2 \mathrm{~cm}$ ). None of the participants had previous experience in NW practice, and none of them deliberately trained for NW out of the learning sessions.

Inclusion criteria included no history of lower extremity injuries within the last 6 months, no previous use of foot orthoses, and being physically active (exercise practice 3 times a week for at least $30 \mathrm{~min}$ per session). All participants gave written informed consent before the commencement of the experiment. The study procedures complied with the Declaration of Helsinki and were approved by the Ethical Committee of the San Antonio Catholic University [TC/02-12].

\section{Measures}

Plantar pressure was recorded with a Biofoot2001® in-shoe pressure measurement system (IBV, Valencia, Spain). This system had been shown to be reliable (Martínez-Nova et al., 2007) and comprises a pair of instrumented insoles each with 64 piezoelectric sensors connected to a logger attached to the waist (Figure $1)$.

The plantar surface was divided into the same seven areas as described for plantar pressure distribution evaluation. The areas used to analyze plantar pressure were adjusted proportionally to the width and length of the foot (for every size of the insole and subject) and were based on the Menz and Morris's scheme (2006). Figure 1 shows the seven anatomical plantar zones used for data analysis (modified from Hessert et al., 2005): hallux (Ha), toes (T), medial metatarsal (MM; first metatarsal head), central metatarsal $(\mathrm{CM}$; second and third metatarsal heads), lateral metatarsal (fourth and fifth metatarsals), arch (Ar) and calcaneus (Ca). Previous studies had demonstrated no differences in pressure values between medial and lateral arch zones, and medial and lateral calcaneus zones during Nordic walking practice (PérezSoriano et al., 2011), thus we decided to join the zones.

Mean peak pressure was calculated as a dependent variable and it was defined as the average value of the maximum pressure from each step recorded over the foot region analyzed (Lucas-Cuevas et al., 2014). Plantar pressure was recorded at $750 \mathrm{~Hz}$ in the right foot. Before measurements, researchers performed a static and dynamic calibration of the plantar pressure system following the manufacturer's instructions to ensure the correct system operation. Trials that were outside the $5 \%$ allowed (faster or slower) target speed were discarded and had to be repeated. The speed was controlled by two photocells (Velleman PEM10D®) connected to an electronic timer (Chronopic, Chronojump BoscoSystem $\left.{ }^{\circledR}\right)$ and located five meters apart from each other. Three steps per trial were selected for analysis. These steps needed to be between the photocells to ensure that the measured speed corresponded to the mean speed at which the steps were performed. In selecting the steps, the experimenter started the pressure measurement, counted the steps and noted the first and last step taken between the photocells.

\section{Procedures}

Prior to the testing session, participants made five familiarization sessions of $1.5 \mathrm{~h}$ each $(7.5 \mathrm{~h}$ of practice) in order to learn the corresponding technique according with the randomly assigned group. Familiarization sessions consisted of technical teaching sessions where participants were instructed how to perform each NW technique according to the International Nordic Walking Association (INWA) technical model for the DT group, and the INFO'-ANE for the AT group. All sessions were supervised by a sports science professional and an experienced instructor of NW. During the NW test, participants of the DT group walked with the trunk bent slightly forward according with the technique pattern described by the INWA; and the AT group walked with the trunk more upright following INFO'-ANE's recommendation. On the other hand, participants walked with their normal walking pattern during the $\mathrm{W}$ test.

At the experimental trials, participants performed a standardized 5 min warm-up at submaximal aerobic speed of NW followed by some stretching and technical exercises. Afterwards, participants walked along a $20 \mathrm{~m}$ long $\times 1.5 \mathrm{~m}$ wide walkway at two speed conditions: freely chosen speed (preferred speed), and fast speed (fast speed $=$ preferred plus 20\%) (Pérez-Soriano et al., 2011). To calculate the preferred condition before the testing session, participants were asked to walk for ten times at a preferred to vigorous intensity for both gait conditions (NW and W). 
Then, we discarded the fastest and the slowest repetition and calculated the mean for each speed condition. Once participants were familiar with the testing procedure, five trials for both NW and $\mathrm{W}$ at the different walking speeds were performed in random order (Figure 2). Participants rested for two minutes between repetitions.

A trial was considered valid when a complete foot strike was captured by the two photocells and the walking speed fell within a 5\% deviation range of the trial speed.

\section{Statistical Analysis}

The SPSS.19® statistical package was used for statistical analyses. After checking the normality of the variables (a Shapiro-Wilk test), a descriptive analysis of the data was performed. The sphericity assumption was verified by the Mauchly test. Then a Two-Way Repeated Measures ANOVA with speed (preferred, fast) and the gait (NW, W) as within-subject factors and plantar pressure as the dependent variable was carried out. We also put as the betweensubject factor the group of technique to know the differences between techniques. After that, a t-test for independent measures was used to identify the specific differences between NW techniques (DT vs. AT). To provide meaningful analysis for comparisons, the effect sizes (ES) were calculated (Cohen's d). Cohen's d was calculated by dividing the mean difference within groups by the root mean square of both standard deviations. The strength of the effect size was determined as small $(<0.50)$, moderate $(0.50-0.79)$, or large $(>0.80)$ (Cohen, 1988). Significance was set at $\alpha=0.05$. Results are presented as means and standard deviations (SD).

\section{Results}

Results are presented in Tables 1 and 2 . Normality $(P=.467)$, homoskedasticity $(P=.380)$ and sphericity $(P=.225)$ were verified.

Walking speed was significantly higher during NW (Preferred: $1.89 \pm 0.18 \mathrm{~m} / \mathrm{s}$; Fast: $2.17 \pm$ $0.21 \mathrm{~m} / \mathrm{s}$ ) compared to $\mathrm{W}$ (Preferred: $1.76 \pm 0.15$ $\mathrm{m} / \mathrm{s}$; Fast: $2.04 \pm 0.19 \mathrm{~m} / \mathrm{s}$ ) in both speed conditions (mean increase of $7.3 \%, p<0.01$ ).

The repeated measures ANOVA showed significant differences in plantar pressure depending on the speed (Preferred vs. Fast) and type of walk (W vs. NW) (Table 2).

During $\mathrm{W}$, increasing the speed (Fast vs. Preferred) led to greater pressure under calcaneus (Ca) $(\mathrm{F}=15.91, p=0.004$, ES $=0.846)$ and hallux $(\mathrm{Ha})(\mathrm{F}=21.264, p=0.001$, $\mathrm{ES}=1.730)$ as well as to lower pressure under central $(\mathrm{CM})(\mathrm{F}=16.626, p=$ 0.002 , ES $=0.968)$ and lateral metatarsal $(\mathrm{LM})(\mathrm{F}=$ 7.403, $p=0.022$, ES = 0.677) (Table 1).

Results showed no differences in plantar pressure between groups for any speed in $\mathrm{W}$ condition.

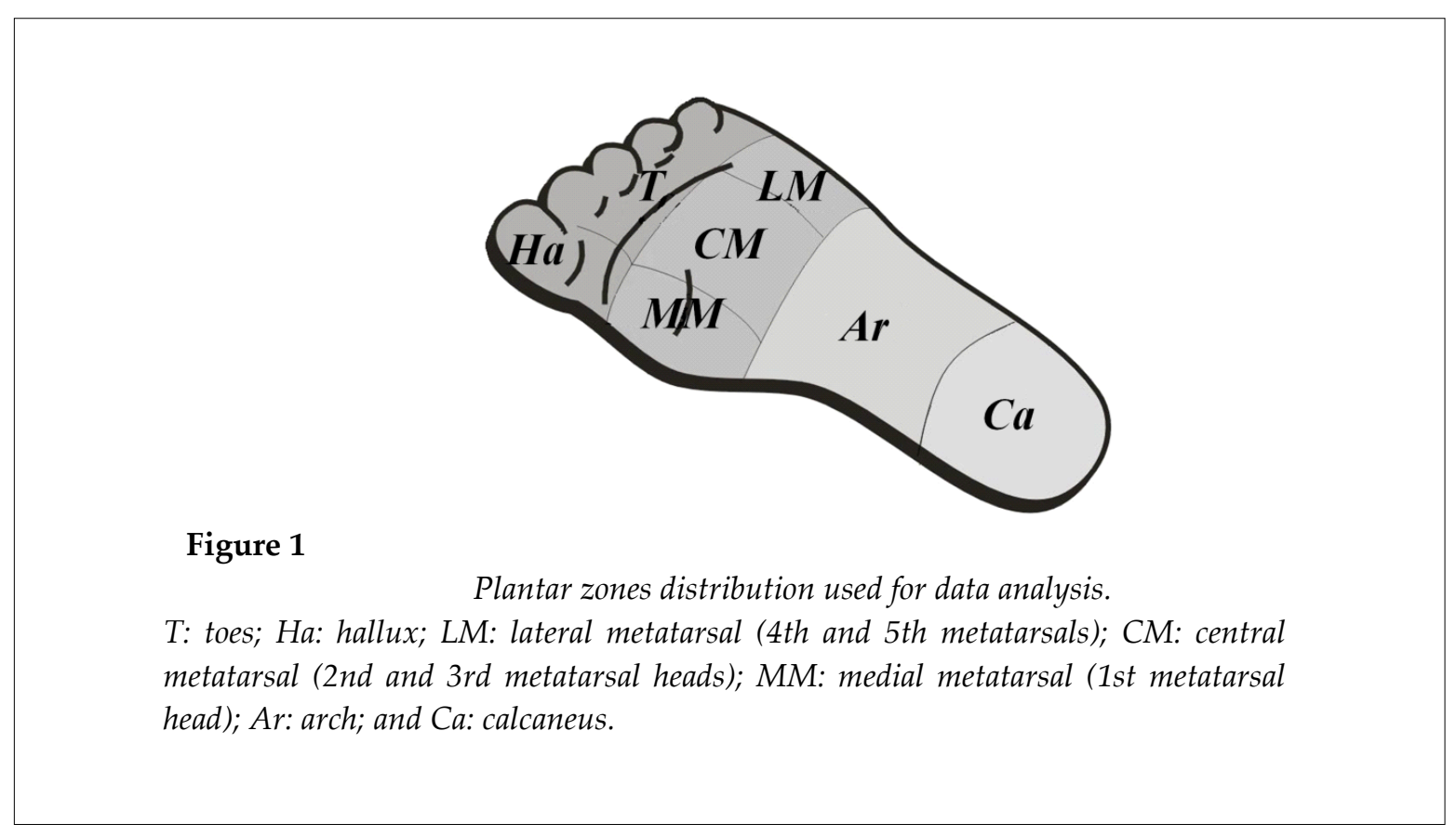




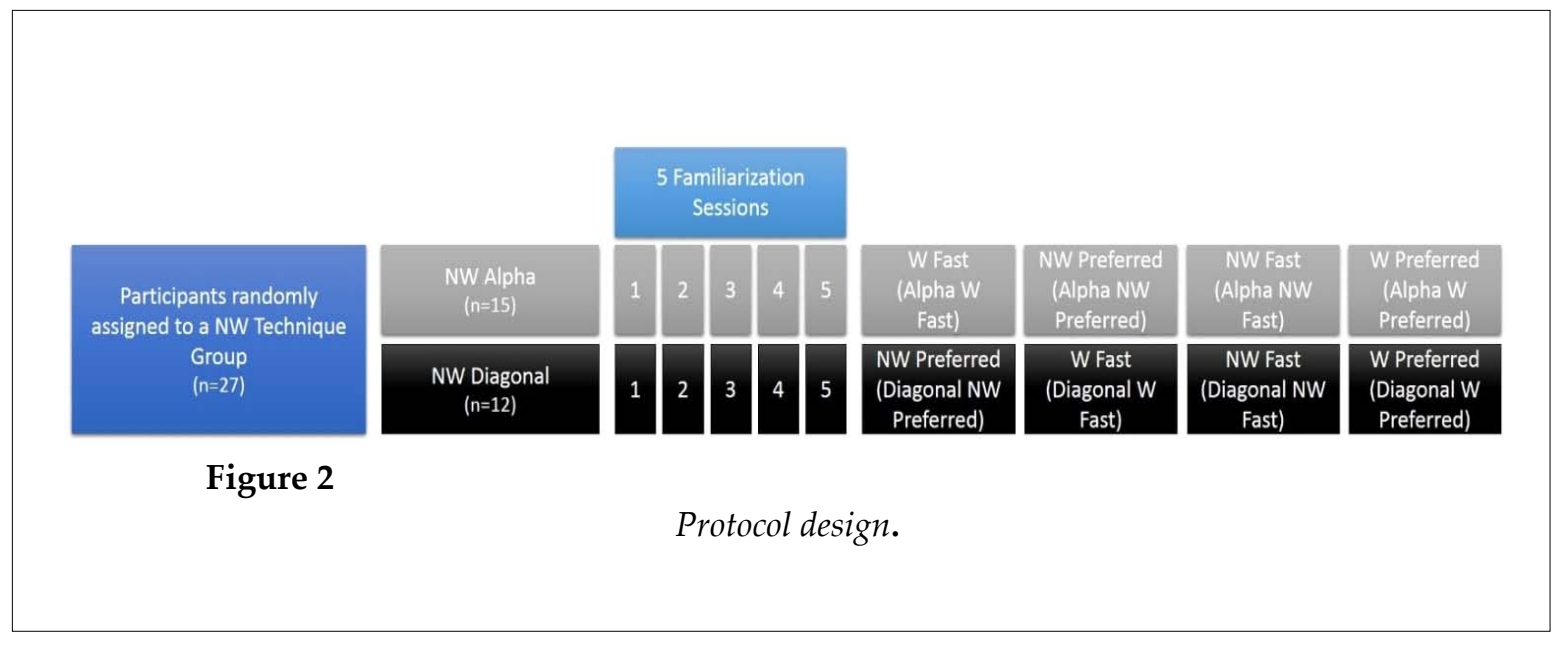

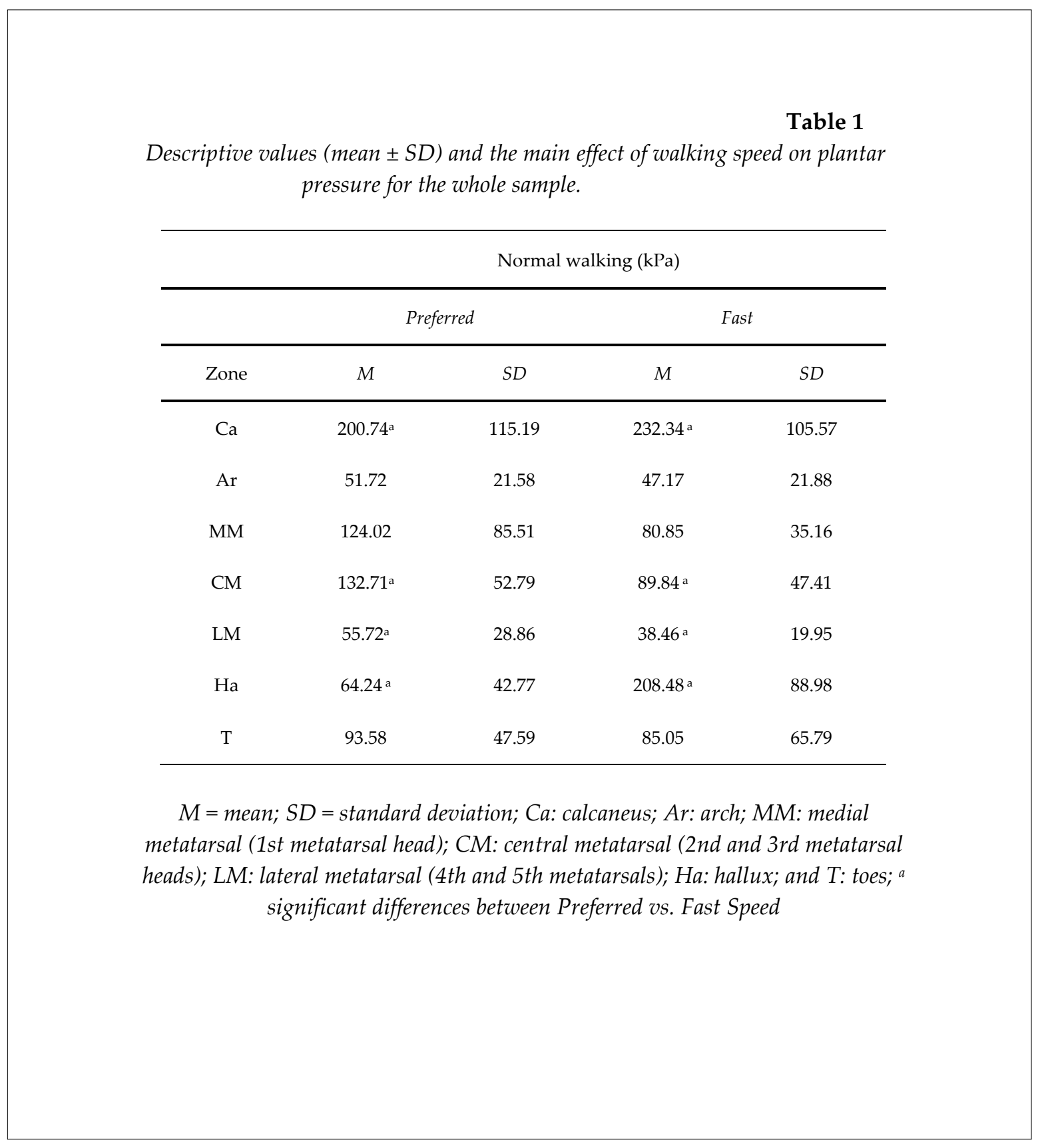




\section{Table 2}

Descriptive values (mean $\pm S D$ ) and the main effect of the gait (Normal walking vs. Nordic walking) on plantar pressure.

\begin{tabular}{ccccc}
\hline & \multicolumn{2}{c}{ Normal walking $(\mathrm{kPa})$} & \multicolumn{2}{c}{ Nordic walking $(\mathrm{kPa})$} \\
\hline Zone & $M$ & $S D$ & $M$ & $S D$ \\
\hline $\mathrm{Ca}$ & 216.54 & 110.38 & 228.50 & 103.96 \\
$\mathrm{Ar}$ & 49.44 & 21.73 & 46.96 & 37.79 \\
$\mathrm{MM}$ & 102.43 & 60.34 & 69.66 & 60.29 \\
$\mathrm{CM}$ & $111.27^{\mathrm{a}}$ & 50.10 & $59.90^{\mathrm{a}}$ & 39.88 \\
$\mathrm{LM}$ & 47.09 & 24.40 & 33.60 & 18.52 \\
$\mathrm{Ha}$ & $136.36^{\mathrm{a}}$ & 65.87 & $48.23^{\mathrm{a}}$ & 36.23 \\
$\mathrm{~T}$ & 89.32 & 56.69 & 74.78 & 45.53
\end{tabular}

$M=\overline{\text { mean; } S D=\text { standard deviation; Ca: calcaneus; Ar: arch; MM: medial metatarsal }}$ (1st metatarsal head); CM: central metatarsal (2nd and 3rd metatarsal heads); LM: lateral metatarsal (4th and 5th metatarsals); Ha: hallux; and T: toes; a significant differences between Walking vs. Nordic walking.

Table 3

Descriptive values (mean $\pm S D$ ) and the main effect of NW technique (AT vs. DT) on plantar pressure.

\begin{tabular}{|c|c|c|c|c|}
\hline \multicolumn{5}{|c|}{ Nordic walking $(\mathrm{kPa})$} \\
\hline \multirow[b]{2}{*}{ Zone } & \multicolumn{2}{|c|}{ Alpha Technique } & \multicolumn{2}{|c|}{ Diagonal Technique } \\
\hline & $M$ & $S D$ & $M$ & $S D$ \\
\hline $\mathrm{Ca}$ & $118.47^{\mathrm{a}}$ & 16,08 & $214.20^{\mathrm{a}}$ & 94,88 \\
\hline $\mathrm{Ar}$ & 28,52 & 21,99 & 39,12 & 37,89 \\
\hline $\mathrm{MM}$ & 34,47 & 43,30 & 69,77 & 42,53 \\
\hline $\mathrm{CM}$ & 38,44 & 21,28 & 87,06 & 64,09 \\
\hline $\mathrm{LM}$ & $21.41^{\mathrm{a}}$ & 18,39 & $50.17^{a}$ & 21,10 \\
\hline $\mathrm{Ha}$ & 35,20 & 30,03 & 66,19 & 50,70 \\
\hline $\mathrm{T}$ & $25,24^{\mathrm{a}}$ & 19,58 & $75,37^{a}$ & 28,86 \\
\hline
\end{tabular}

$M=$ mean; SD = standard deviation; Ca: calcaneus; Ar: arch; MM: medial metatarsal (1st metatarsal head); CM: central metatarsal (2nd and 3rd metatarsal heads); LM: lateral metatarsal (4th and 5th metatarsals); Ha: hallux; and T: toes; ${ }^{a}$ significant differences between AT vs. DT. 
Both technique groups (AT, DT) showed lower pressure during NW compared to $\mathrm{W}$ under Ha at Fast speed $(78.1 \%$ mean descent, $\mathrm{F}=58.321$, $p=0.000$, ES $=2.449)$ and under $\mathrm{CM}$ at both speed conditions $(46.2 \%$ mean descent, $\mathrm{F}=41.917, p=$ 0.012, ES = 1.365) (Table 2).

Moreover, the AT group experienced significantly lower pressure during NW compared to $\mathrm{W}$ under toes (T) at Preferred speed $(\mathrm{F}=11.998, p=0.007, \mathrm{ES}=1.303)$ and under $\mathrm{LM}$ at Fast speed (28.2\% mean descent, $\mathrm{F}=32.246, p=$ 0.048, ES $=0.488$ )

An interaction effect between speed (Preferred vs. Fast) and the type of walk (W and $\mathrm{NW})$ was observed for the $\mathrm{CM}$ and $\mathrm{HA}$ zones $(\mathrm{F}=$ $6.172, p=0.027 ; \mathrm{F}=40.702, p=0.000$; respectively), indicating the existence of different behaviors depending on the condition.

Finally, significant differences were also observed between the NW techniques (Table 3). The AT group experienced significantly lower pressure under $\mathrm{Ca}(44.7 \%$ mean descent, $p=0.046$, $\mathrm{ES}=1.49)$, LM (57.3\% mean descent, $p=0.015$, ES $=1.44)$ and $\mathrm{T}(190 \%$ mean descent, $p=0.040$, ES $=$ 1.20) compared to the DT group at the Preferred speed condition. No differences were observed between techniques at the Fast speed condition.

\section{Discussion}

NW is an activity of increasing popularity due to its accessibility and feasibility (Tschentscher et al., 2013). The upper body activity has been observed to enhance oxygen uptake, increase caloric expenditure and the heart rate, lower the individual's psychological stress, anxiety, depression and the sensations of pain without increasing perceived exertion compared to normal walking (Breyer et al., 2010; Church et al., 2002; Oakley et al., 2008). Moreover, since NW involves the use of about $90 \%$ of the body's muscles (Sentinelli et al., 2015), its practice provides a greater physiological demand and therefore a greater training stimulus compared to $\mathrm{W}$, thereby allowing special populations to increase their levels of physical activity and improve health (Dechman et al., 2011).

However, even though NW has been appointed to be a safe and easy activity for the majority of the population, some training is needed in order to perform the technique correctly. In this regard, little is known about how the different techniques used in NW influence plantar pressure during locomotion. Therefore, the objective of the present study was to compare the plantar pressure loading between different NW techniques (Alpha, Diagonal) and $\mathrm{W}$ at different gait speeds.

Based on previous studies, it was hypothesized that increases in speed would lead to greater plantar pressure. The results observed in the present study partly confirmed this hypothesis. Interestingly, walking faster led to increased pressure under the calcaneus and hallux as well as to reduced pressure under the metatarsals. These results are in agreement with previous studies where greater pressure under the hallux and heel (Burnfield et al., 2004; Drerup et al., 2008; Segal et al., 2004) and lower pressure under the metatarsals (Pérez-Soriano et al., 2011) were observed as the speed increased. This behavior of the plantar loading could be due to the increased speed at which the foot proceeds from the heel strike to toe-off, reducing the time the individual places weight on the forefoot and resulting in greater forces only at the hallux during toe-off (Pérez-Soriano et al., 2011; Segal et al., 2004). Moreover, both speed conditions (Preferred, Fast) were faster during NW than during $\mathrm{W}$. This result indicates that an individual walks faster with poles both at a preferred and fast (self-selected) pace compared to walking without poles, increasing the energy expenditure and experiencing a stronger training stimulus without increasing the plantar pressure.

Secondly, it was also hypothesized that NW would reduce plantar pressure compared to W. The results of this study support this hypothesis since the NW Alpha reduced plantar pressure under the hallux $(83 \%)$, toes $(77 \%)$ as well as central $(68 \%)$ and lateral metatarsals $(28 \%)$ compared to W, whereas NW Diagonal reduced plantar pressure under the hallux $(73 \%)$ and central metatarsals (39\%) compared to W. This reduction could be due to the function of the pole as an additional point of support compared to $\mathrm{W}$ where all the loading is experienced and absorbed through the plantar surface (Kanade et al., 2006; Pérez-Soriano et al., 2011).

The reduction in plantar pressure under the metatarsals and hallux is of great relevance due to the purported deleterious effects of excessive pressure under these areas in certain 
populations with frail feet such as individuals with rheumatoid arthritis, obesity, or diabetes mellitus (van der Leeden et al., 2008). Previous studies have observed that in patients with the diabetic neuropathic foot, elevated plantar pressure under the metatarsals is usually observed (Mueller et al., 2005; Van Shie and Boulton, 2006). Moreover, excessive plantar pressure has been suggested as a cause of the development of plantar ulcers (Pham et al., 2000), and ulceration is often a precursor of lower extremity amputation (Pecorato et al., 1990). On the other hand, other authors have also identified that hallux valgus and hallux rigidus patients experience elevated pressure under the hallux and suggested that this elevated loading may even represent a pathological status and demonstrate a worse clinical picture (Martínez-Nova et al., 2010). Taking into account these observations, relieving between $28-83 \%$ of the pressure experienced under these areas by practicing NW may imply an important benefit for these populations. High plantar pressure associated with some biomechanical adaptations indicates the need of new strategies that would contribute to reducing this pressure by means of exercise, especially in some populations like obese people who experience an increment in plantar pressure under several regions of the foot (Castro et al., 2014), older people in whom it has been demonstrated that high plantar pressure generated during the gait may contribute to foot pain and risk of falls (Mickle et al., 2010), and in women at the third trimestral of pregnancy that experience an increase of pressure at forefoot regions (Karadagan-Saygi et al., 2010).

Finally, it was also hypothesized that the different NW techniques would lead to significant changes in plantar pressure. Indeed, the results of the present study support this hypothesis since NW Alpha reduced plantar pressure under the calcaneus $(45 \%)$, lateral metatarsals $(57 \%)$, and toes $(67 \%)$ compared to NW Diagonal. It is believed that the differences in plantar pressure may be explained by differences in the mechanics between the two techniques. In this sense, whereas the DT is strongly influenced by crosscountry skiing and therefore the athletes tend to bend their trunk slightly forward in order to achieve greater speed with longer strides, the AT is based on a more vertical position of the trunk throughout the movement (Martinez-Lemos, 2010). The position of the trunk during NW Diagonal may allow for a greater stride length, where the foot would contact the ground with greater ankle dorsiflexion and the heel would experience a greater and more vertical impact during ground contact, similar to race walking (Elvira et al., 2008). On the other hand, due to the upright position of the trunk during NW Alpha, the center of gravity would be closer to the trunk compared to NW Diagonal and this would make the foot contact time shorter in a position in which the subjects apply anterior-posterior braking forces that are directly correlated with greater plantar pressure under the heel (Allet et al., 2011). Even though this is pure speculation at this point and the mechanical differences between techniques remain unknown from a scientific perspective, future studies should carry out kinematic analysis of the different NW techniques in order to objectively support these hypotheses. Despite the differences, both NW techniques significantly reduce plantar pressure, though the characteristics of the Alfa technique make it more appropriate for risk populations such as diabetics, obese, pregnant women and the elderly because the higher pressure in such populations is registered in the areas where greater pressure reductions are observed compared with the Diagonal technique (heel, lateral metatarsals and toes). Although we allowed the participants to choose their walking speed in order to recreate a more natural walking pattern, differences in walking speed influenced plantar pressure and it could be thus considered a study limitation. However, greater speed was observed during both NW conditions (Alpha, Diagonal) compared to $\mathrm{W}$, thereby implying that the pressure reduction observed during NW would be even greater if all gait conditions had been measured at the same walking speed.

\section{Conclusions}

Our data shows that Nordic Walking practice reduces plantar pressure distribution compared with Walking in every speed condition, and NW Alpha Technique seems to be the most appropriate in order to reduce plantar pressure.

For practical purposes, the results of this study will be of benefit to the practitioners seeking the healthiest Nordic Walking technique 
in terms of load reduction on feet. It could also be helpful for coaches and conditioning professionals to use the most appropriate technique depending on the population with whom they will be working, adapting the workload depending on the characteristics of practitioners, as well as selecting the best technique to reduce the injury risk in sensitive populations. Similar studies should be conducted on special populations.

\section{Acknowledgments:}

This study was supported by the Catholic University of Murcia (Spain) [TC/02-12] under an internal project awarded to the Research Group on Health, Physical Activity, Fitness and Motor Behavior (GISAFFCOM).

\section{References}

Allet L, IJzerman H, Meijer K, Willems P, Savelberg H. The influence of stride-length on plantar footpressures and joint moments. Gait Posture, 2011; 34(3): 300-306

Breyer MK, Breyer-Kohansal R, Funk GC, Dornhofer N, Spruit MA, Wouters E, Burghuber OC, Hartl S. Nordic walking improves daily physical activities in COPD: a randomised controlled trial. Respir Res, 2010; 11(1): 112

Brunelle EA, Miller MK. The effects of walking poles on ground reaction forces. Res Q Exerc Sport, 1998; 69(Suppl.): A30

Burnfield JM, Few CD, Mohamed OS, Perry J. The influence of walking speed and footwear on plantar pressures in older adults. Clin Biomech, 2004; 19(1): 78-84

Church TS, Earnest CP, Morss GM. Field testing of physiological responses associated with Nordic Walking. Res Q Exerc Sport, 2002; 73: 296-300

de Castro MP, Abreu SC, Sousa H, Machado L, Santos R, Vilas-Boas JP. In-Shoe Plantar Pressures and Ground Reaction Forces During Overweight Adults' Overground Walking, Res Q Exerc Sport, 2014; 85(2): 188-197

Dechman G, Appleby J, Carr M, Haire M. Comparison of treadmill and over-ground Nordic walking. Eur J Spost Sci, 2012; 12(1): 36-42

Drerup B, Szczepaniak A, Wetz HH. Plantar pressure reduction in step-to gait: A biomechanical investigation and clinical feasibility study. Clin Biomech, 2008; 23: 1073-1079

Elvira JLL, Vera-García FJ, Meana M, García JA. Biomechanical analysis of plantar support in race walking. Relationship between the footprint, angles of the subtalar joint and plantar pressures. Mot Eur J Hum Mov, 2008; 20: 41-60

Encarnación-Martínez A, Pérez-Soriano P, Llana-Belloch S. Differences in ground reaction forces and shock impacts between nordic walking and walking. Res $Q$ Exerc Sport, 2015; 86(1): 94-99

Figard-Fabre H, Fabre N, Leonardi A, Schena F. Physiological and perceptual responses to Nordic walking in obese middle-aged women in comparison with the normal walk. Eur J Appl Physiol, 2010; 108(6): $1141-1151$

Hagen M, Henning EM, Stieldorf P. Lower and upper extremity loading in nordic walking in comparison with walking and running. J Appl Biomech, 2011; 27: 22-31

Hudson D. The effect of walking with poles on the distribution of plantar pressures in normal subjects. PMER, 2014; 6(2): 146-151

Kanade RV, van Deursen RW, Harding K, Price P. Walking performance in people with diabetic neuropathy: 
Benefits and threats. Diabetologia, 2006; 49: 1747-1754

Karadag-Saygi E, Unlu-Ozkan F, Basgul A. Plantar pressure and foot pain in the last trimester of pregnancy. Foot Ankle Inte, 2010; 31(2): 153-157

Lucas-Cuevas AG, Pérez-Soriano P, Llana-Belloch S, Macián-Romero C, Sánchez-Zuriaga D. Effect of custom-made and prefabricated insoles on plantar loading parameters during running with and without fatigue. J Sports Sci, 2014; 32(18): 1712-1721

Lyons TE, Rosenblum BI, Veves A. Foot pressure abnormalities in the diabetic foot. In The Diabetic Foot, 2012; 255-274, Humana Press

Martínez-Lemos RI. Alfa247®: A model of differentiated learning for teaching Nordic Walking. Rev Investig Educ, 2010; 7: 123-130

Martínez-Nova A, Cuevas-García J, Pascual-Huerta J, Sánchez-Rodríguez R. BioFoot ${ }^{\circledR}$ in-shoe system: Normal values and assessment of the reliability and repeatability. Foot, 2007; 17(4): 190-196

Martínez-Nova A, Sánchez-Rodríguez R, Pérez-Soriano P, Llana-Belloch S, Leal Muro A, Pedrera-Zamorano JD. Plantar pressures determinants in mild hallux valgus. Gait Posture, 2010; 32: 425-427

Menz HB, Morris ME. Clinical determinants of plantar forces and pressures during walking in older people. Gait Posture, 2006; 24(2): 229-236

Mickle KJ, Munro BJ, Lord SR, Menz HB, Steele JR. Foot pain, plantar pressures, and falls in older people: a prospective study. J Amer Geriat Soc, 2010; 58(10): 1936-1940

Mueller MJ, Zou D, Lott DJ. Pressure gradient as an indicator of plantar skin injury. Diabetes Care, 2005; 28: 2908-2912

Oakley C, Zwierska I, Tew G, Beard JD, Saxton JM. Nordic poles immediately improve walking distance in patients with intermittent claudication. Eur J Vas Endovasc, 2008; 36(6): 689-694

Pecoraro RE, Reiber GE, Burgess EM. Pathways to diabetic limb amputation. Basis for prevention. Diabetes Care, 1990; 13: 513-521

Pérez-Soriano P, Encarnación-Martínez A, Aparicio-Aparicio I, Giménez JV, Llana-Belloch S. Nordic Walking: a systematic review. Eur J Hum Mov, 2014; 33: 26-45

Pérez-Soriano P, Llana-Belloch S, Martínez-Nova A, Morey G, Encarnación-Martínez A. Nordic walking practice might improve plantar pressure distribution. Res Q Exerc Sport, 2011; 82(4): 593-599

Perry JE, Ulbrecht JS, Derr JA, Cavanagh PR. The use of running shoes to reduce plantar pressures in patients who have diabetes. J Bone Joint Surg Am, 1995; 77(12): 1819-1828

Pham H, Armstrong DA, Harvey C, Harkless LB, Giurini JM, Veves A. Screening techniques to identify people at high risk for diabetic foot ulceration. Diabetes Care, 2000; 23: 606-611

Schwameder H, Ring S. Knee joint loading and metabolic energy demand in walking, nordic walking and running. J Biomech, 2006; 39(Suppl.1): S185

Segal A, Rohr E, Orendurff M, Shofer J, O'Brien M, Sangeorzan B. The effect of walking speed on peak plantar pressure. Foot Ankle Int, 2004; 25: 926-933

Sentinelli F, La Cava V, Serpe R, Boi A, Incani M, Manconi E, Solinas A, Cossu E, Lenzi A, Baroni MG. Positive effects of Nordic Walking on anthropometric and metabolic variables in women with type 2 diabetes mellitus. Sci Sport, 2015; 30(1): 25-32

Stief F, Kleindienst FI, Wiemeyer J, Wedel F, Campe S, Krabbe B. Inverse dynamic analysis of the lower extremities during nordic walking, walking, and running. J Appl Biomech, 2008; 24(4): 351-359

Tschentscher M, Niederseer D, Niebauer J. Health benefits of Nordic walking: a systematic review. Am J Prev 
Med, 2013; 44(1): 76-84

van der Leeden M, Steultjens MP, Terwee CB, Rosenbaum D, Turner D, Woodburn J, Dekker J. A systematic review of instruments measuring foot function, foot pain, and foot-related disability in patients with rheumatoid arthritis. Arthritis Rheum, 2008; 59: 1257-1269

Van Shie CHM, Boulton AJM. Biomechanics of the diabetic foot. The diabetic foot (2nd ed). Totowa: Humana Press, 186-200; 2006

Vela SA, Lavery LA, Armstrong DG, Anaim AA. The effect of increased weight on peak pressures: implications for obesity and diabetic foot pathology. Foot Ankle Surg, 1998; 37(5): 416-420

\title{
Corresponding author:
}

\author{
Alberto Encarnación-Martínez, PhD \\ Catholic University of Murcia \\ Department of Sport and Physical Education, \\ Campus de Los Jerónimos, s/n \\ 30107 Guadalupe, Spain. \\ Tel: +34 968278824 \\ Fax: +34 968278655 \\ E-mail: aencarnacion@ucam.edu
}

